\title{
Thermoinduced magnetic moment in akaganéite nanoparticles
}

\author{
A. Urtizberea, ${ }^{1}$ F. Luis, ${ }^{1,{ }^{*}}$ A. Millán, ${ }^{1}$ E. Natividad,${ }^{2}$ F. Palacio, ${ }^{1}$ E. Kampert,${ }^{3}$ and U. Zeitler ${ }^{3}$ \\ ${ }^{1}$ Instituto de Ciencia de Materiales de Aragón (ICMA), CSIC and Departamento de Física de la Materia Condensada, Facultad de Ciencias, \\ Universidad de Zaragoza, E-50009 Zaragoza, Spain \\ ${ }^{2}$ Instituto de Ciencia de Materiales de Aragón (ICMA), CSIC and Departamento de Ciencia y Tecnología de Materiales y Fluidos, Campus \\ Río Ebro, Edificio Torres Quevedo, Universidad de Zaragoza, E-50018 Zaragoza, Spain \\ ${ }^{3}$ Radboud University Nijmegen, Institute for Molecules and Materials, High Field Magnet Laboratory, Toernooiveld 7 , \\ NL-6525 ED Nijmegen, The Netherlands \\ (Received 24 June 2010; revised manuscript received 19 April 2011; published 29 June 2011)
}

\begin{abstract}
It is shown that akaganéite $\beta$-FeOOH provides a good model material to experimentally investigate thermoinduced magnetic moments in antiferromagnetic nanoparticles. We characterize the magnetic properties, exchange field, anisotropy field, and antiferromagnetic susceptibility of bulk akaganéite. In the nanoparticles, we find a drastic enhancement of the antiferromagnetic susceptibility, a phenomenon first predicted by Néel. Also, we find that akaganéite nanoparticles possess a thermoinduced magnetic moment.
\end{abstract}

DOI: 10.1103/PhysRevB.83.214426

PACS number(s): 75.75.-c, 75.30.Ds, 75.50.Ee, 75.50.Tt

\section{INTRODUCTION}

Antiferromagnetic nanoparticles exhibit a number of interesting size-dependent phenomena, not present in particles of ferromagnetic materials. Some of them were already discussed by Néel in his early works. ${ }^{1-3}$ The antiferromagnetic susceptibility, that is, the continuous rotation of each sublattice magnetization toward a magnetic field perpendicular to the anisotropy axis, becomes enhanced in nanoparticles with respect to the bulk. ${ }^{2-5}$ Another feature arises from the decompensation of surface spins that confers a net magnetic moment to antiferromagnetic nanoparticles. ${ }^{1,6,7}$ Recently, a new interesting phenomenon concerning antiferromagnetic nanoparticles has been predicted to occur at finite temperatures, ${ }^{8}$ based on the particular character of antiferromagnetic spin waves. ${ }^{9,10}$ In a uniform spin-wave mode of frequency $\omega_{0}$ the two sublattices precess with different amplitudes, and the angle between them increases with the excitation energy. The thermal population of this mode should then lead to an uncompensated magnetic moment that, in contrast to the one associated with surface disorder, would increase with temperature and would be independent of the nanoparticle's size. In bulk materials, inhomogeneous spin-wave modes are also populated and, consequently, the thermoinduced magnetic moment should not be noticeable. In antiferromagnetic nanoparticles, by contrast, the number of allowed inhomogeneous modes is reduced and their energies increased, thereby making the thermoinduced contribution appreciable.

Some authors have reported experimental evidence of this phenomenon in nanoparticles of ferrihydrite. ${ }^{4,6,11}$ However, the increase with temperature of the magnetic moment found in these studies has been questioned. The magnetic moment was obtained from magnetization isotherms, neglecting the influence of the particle size distribution ${ }^{12}$ and magnetic anisotropy. ${ }^{13,14}$ Another experimental difficulty is that the intrinsic magnetic properties of ferrihydrite are not well known because it cannot be produced as a bulk material.

Akaganéite, $\beta-\mathrm{FeOOH}$, seems to be a more adequate antiferromagnetic model system to investigate those size-dependent phenomena. It can be prepared as nanoparticles as well as bulklike microcrystals. Here, we report a detailed study of the magnetic response of akaganéite in both forms. We have investigated how intrinsic magnetic properties such as the ordering temperature, the effective spin, and the antiferromagnetic susceptibility are affected by the reduction of size. The uncompensated magnetic moment of the nanoparticles was obtained from linear susceptibility data, which are nearly independent of the anisotropy. ${ }^{13,14}$ This experimental approach and the good characterization of akaganéite magnetic properties enable us to determine the thermoinduced magnetic moment.

The paper is organized as follows. Section II describes the synthesis of the samples, while Sec. III provides experimental details of the physical characterization. In Sec. IV we describe the experimental results. First, the intrinsic magnetic properties of the bulk sample are reported, that is, the ordering temperature, the antiferromagnetic susceptibility, and the magnetic anisotropy, which will be useful to explain the results obtained for the nanoparticle's magnetic moment and its physical origin. The magnitudes of these intrinsic magnetic properties are next compared with those of the nanoparticles. Then, the thermoinduced contribution to the nanoparticle magnetic moment is determined from the linear susceptibility. Finally, this contribution is analyzed in terms of the model proposed in Ref. 15. Section V is left for the conclusions.

\section{SAMPLE CHARACTERISTICS}

Akaganéite is the naturally occurring form of $\beta-\mathrm{FeOOH}$. The unit cell is monoclinic (symmetry $I 2 / m$ ). ${ }^{16}$ The structure, shown in Fig. 1, consists of double chains of edge-sharing $\mathrm{Fe}^{3+}-(\mathrm{O}, \mathrm{OH})$ octahedra. These double chains share corners with adjacent chains to give a three-dimensional structure containing channels with square cross sections that measure two octahedra per side. These channels, parallel to the $b$ axis, are partially occupied by chloride anions which confer stability on the crystal structure. The exchange interactions between $\mathrm{Fe}^{3+}$ ions in akaganéite result in an antiferromagnetic 

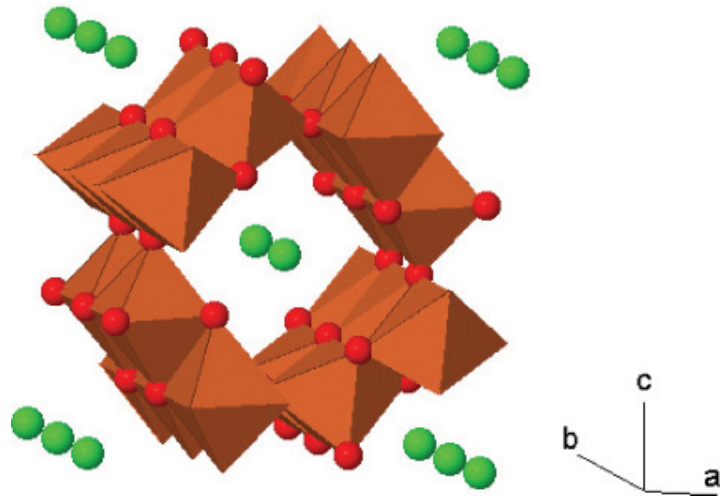

FIG. 1. (Color online) Arrangement of octahedral double chains running parallel to the $b$ axis with $\mathrm{Cl}^{-}$ions in the channels.

(AF) material with a Néel temperature $T_{\mathrm{N}} \sim 240-299$ K. ${ }^{17}$ The magnetic cell, proposed from neutron diffraction studies, coincides with the chemical unit cell such that the spins interact antiferromagnetically and are oriented along the $b$ axis. ${ }^{18}$ This model was later supported by the results of Mössbauer experiments performed on oriented particles. $^{19}$

The synthesis and physical characterization of the samples we used in this work are described in Ref. 20. The bulk akaganéite sample was prepared by the spontaneous oxidative hydrolysis of $\mathrm{FeCl}_{2}$ solutions. Akaganéite nanoparticles were prepared using a polyvinyl-pyridine polymer matrix as a mold in order to control the particle size and particle size dispersion, and to prevent aggregation. Both samples were investigated by $x$-ray powder diffraction while the latter sample was also investigated by high-resolution transmission electron microscopy. From these measurements we conclude that the only iron oxide phase present in the samples is akaganéite. ${ }^{20}$ Scanning electron microscopy (SEM) observations showed that the bulk sample consists of elongated particles with an average length of $33 \mu \mathrm{m}$ and an average width of $2.5 \mu \mathrm{m}$. A typical SEM image is shown in Fig. 2(a). Transmission electron microscopy (TEM) images of this sample allow us to discard the presence of any appreciable fraction of particles with sizes in the nanometer range. The nanocomposite sample corresponds to the one defined in Ref. 20 as $\mathrm{NC}_{\mathrm{Cl}}$. The size distribution and morphology of the nanoparticles were determined by TEM. A representative image is shown in Fig. 2(b). The nanoparticles are rodlike with an average length of $18 \mathrm{~nm}$ and an average width of $5 \mathrm{~nm}$.

\section{EXPERIMENT}

Magnetic measurements were performed using commercial superconducting quantum interference device and vibratingsample magnetometers. High-field (up to $300 \mathrm{kOe}$ ) magnetization curves were also measured, at different temperatures, using an extraction magnetometer in a Bitter magnet at the High Field Magnet Laboratory (HFML) in Nijmegen. ${ }^{21}$ The diamagnetic contributions of the polymer and the sample holder were subtracted from the experimental data.

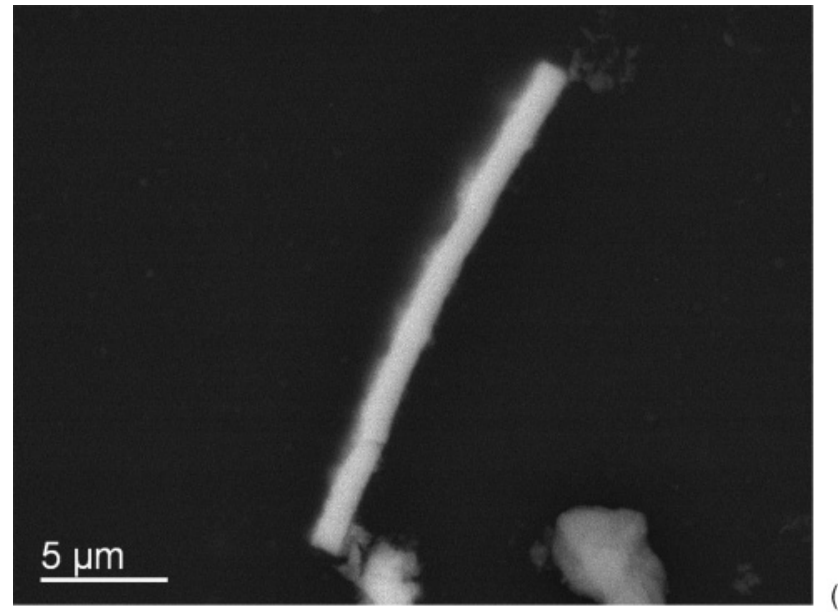

(a)

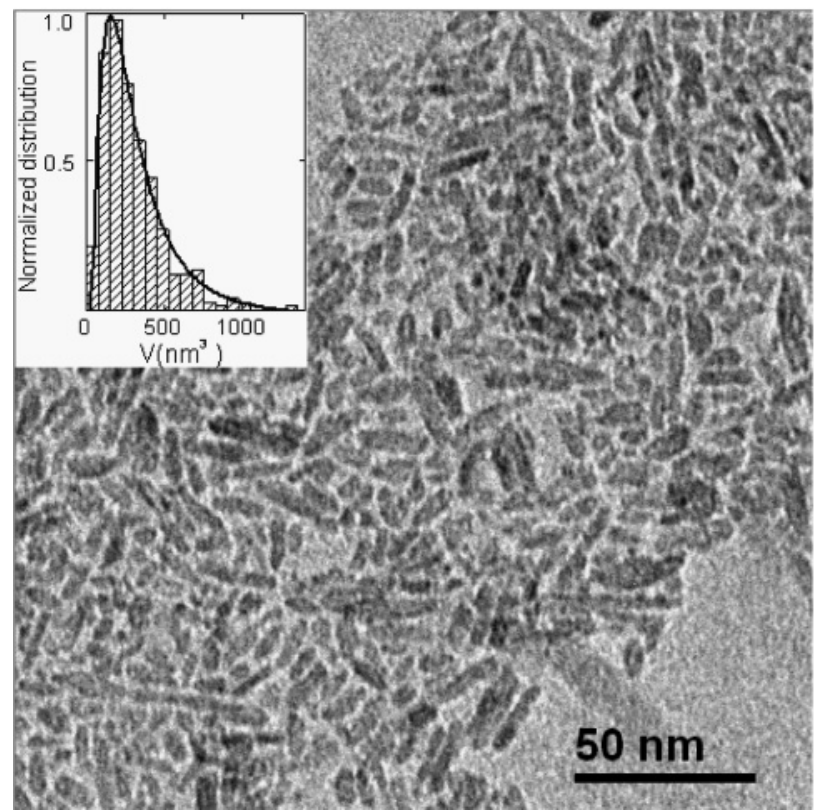

(b)

FIG. 2. SEM and TEM images of the bulk (a) and the nanoparticle (b) samples. Inset: Volume distribution determined by TEM and the corresponding fit to a log-normal function.

\section{RESULTS}

\section{A. Intrinsic magnetic properties}

\section{Bulk}

ac susceptibility data of bulk akaganéite are shown in Fig. 3(a). Above the Néel temperature this susceptibility follows the Curie-Weiss law,

$$
\chi_{\mathrm{AF}}=\frac{N \mu_{\mathrm{eff}}^{2}}{3 k_{\mathrm{B}}(T-\theta)}
$$

where $\theta=-595 \pm 28 \mathrm{~K}$ is the Weiss temperature, and $\mu_{\text {eff }} \equiv$ $\sqrt{g^{2} \mu_{\mathrm{B}}^{2} S(S+1)}=(4.4 \pm 0.2) \mu_{\mathrm{B}}$ is the effective magnetic moment of each $\mathrm{Fe}^{3+}$ ion, with a gyromagnetic ratio $g$ and spin $S$. Using $g=2$ this gives $S=1.75 \pm 0.1$ for the $\mathrm{Fe}^{3+}$ ions. This value of $S$ agrees with values reported in Ref. 17. However, $S$ is lower than $S=5 / 2$ expected from the application of Hund's rules, as observed in other iron oxides. ${ }^{22}$ A possible spin reduction mechanism might be associated 

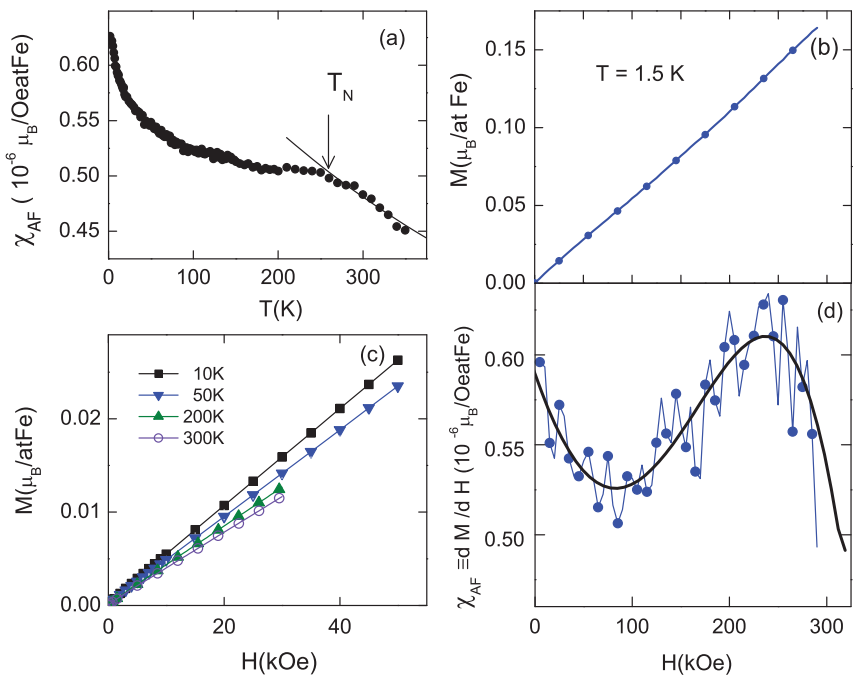

FIG. 3. (Color online) Bulk akaganéite. (a) In-phase susceptibility. The solid line represents the Curie-Weiss law characteristic of the paramagnetic phase $\left(T>T_{\mathrm{N}} \sim 260 \mathrm{~K}\right)$. Magnetization vs field curves at fields $\leqslant 320 \mathrm{kOe}(\mathrm{b})$ and $\leqslant 50 \mathrm{kOe}(\mathrm{c})$. (d) The derivative of the high-field magnetization vs field, showing evidence for a spin-flop transition at $1.5 \mathrm{~K}$. The solid line represents the derivative of the interpolated magnetization data.

with the compression of the coordination octahedra toward the $\mathrm{Fe}^{3+}$ ions by the interstitial ions in the structure. This compression would then enhance the covalency of the $\mathrm{Fe}-\mathrm{O}$ bond and consequently diminish the effective $3 d$ spin. ${ }^{17}$ This mechanism was proposed by Chambaere and De Grave ${ }^{17}$ in order to explain the reduction of the effective spin $S$ observed as the number of interstitial water molecules in the structure was increased.

Below the Néel temperature, the ac susceptibility data agree with the results of previous susceptibility studies of akaganéite. ${ }^{23}$ Notice that $\chi_{\mathrm{AF}}$ does not show a maximum near $T_{\mathrm{N}}$, as expected for a conventional AF material. ${ }^{9}$ The origin of this feature will be discussed below.

The sublattice magnetization is estimated as $M_{\mathrm{S}}=\frac{1}{2} N g \mu_{\mathrm{B}} S=413.6 \mathrm{emu} / \mathrm{cm}^{3}$, where $\quad N=2.5 \times$ $10^{22}$ at. $\mathrm{Fe} / \mathrm{cm}^{3}$ for akaganéite.

The Néel temperature $T_{\mathrm{N}} \approx 260 \mathrm{~K}$ was determined as the temperature where the susceptibility deviates from the Curie-Weiss behavior [see Fig. 3(a)]. This value was confirmed by heat capacity measurements (not shown). The exchange field $H_{\mathrm{E}}$ may be inferred from the Néel temperature, using mean field theory which predicts $T_{\mathrm{N}}=H_{\mathrm{E}} g \mu_{\mathrm{B}}(S+1) / 3 \mathrm{k}_{\mathrm{B}}$, which gives $H_{\mathrm{E}}=2.1 \times 10^{6} \mathrm{Oe}$. In principle, $H_{\mathrm{E}}$ could also be estimated from $\chi\left(T_{\mathrm{N}}\right)=5 \times 10^{-7} \mu_{B} / \mathrm{Oe}$ (at. Fe), using the relation $H_{\mathrm{E}}=M_{\mathrm{S}} / \chi\left(T_{\mathrm{N}}\right)$, which gives $H_{\mathrm{E}}=3.5 \times 10^{6}$ Oe. However, this expression only holds for magnetic structures in which next-nearest neighbor interactions can be neglected. Since the magnetic structure of akaganéite involves secondneighbor interactions (see Ref. 18) we will use the mean field value $H_{\mathrm{E}}=2.1 \times 10^{6}$ Oe here.

The antiferromagnetic susceptibility can also be obtained under finite magnetic fields. For this, we performed the derivative of magnetization curves shown in Figs. 3(b) and 3(c). A closer inspection of the magnetization curves reveals that for $H<100 \mathrm{kOe}, \chi_{\mathrm{AF}}$ decreases with increasing $H$ [Fig. 3(d)]. This effect, and the unexpected temperature dependence of $\chi_{\mathrm{AF}}$ below $T_{\mathrm{N}}$, can be tentatively explained as the result of a weak spin canting in the magnetic structure, which has also been proposed by Barrero et al. ${ }^{24}$ to account for their Mössbauer spectroscopy results. In order to quantify the uncompensated magnetization arising from the spin canting the magnetization curves were extrapolated to zero field, giving $M_{\mathrm{unc}}=3.5 \times 10^{-4} \mu_{\mathrm{B}} /$ at. Fe. Using this value and the effective spin determined above the maximum spin canting angle is estimated $\leqslant 0.002^{\circ}$.

The anisotropy field $H_{\text {an }}$ of bulk akaganéite can be determined by analyzing the spin-flop transition. Some evidence of this transition is provided by the differential susceptibility shown in Fig. 3(d), obtained by differentiating the $M(H)$ data measured at very high fields [Fig. 3(b)]. The susceptibility shows a maximum, centered at a magnetic field $H_{\mathrm{sf}} \sim$ $240 \mathrm{kOe}$, which can be attributed to this transition. The susceptibility maximum is probably smeared out by the random orientation of the anisotropy axis in the powdered sample.

The antiferromagnetic susceptibility of a sample with randomly oriented easy axes $\chi_{\mathrm{AF}}=\frac{1}{3} \chi_{\mathrm{AF} \|}+\frac{2}{3} \chi_{\mathrm{AF} \perp}$, where $\chi_{\mathrm{AF} \|}$ and $\chi_{\mathrm{AF} \perp}$ are the longitudinal and transverse susceptibility components, respectively. At $T \sim 1.5 \mathrm{~K} \chi_{\mathrm{AF}||}$ is expected to be close to zero. ${ }^{9}$ Then, the spin-flop field $H_{\text {sf }}$ is

$$
H_{\mathrm{sf}} \simeq \sqrt{\frac{2 K}{\chi_{\mathrm{AF} \perp}}},
$$

where $K$ is the magnetic anisotropy constant. In order to determine $K$ we use for $\chi_{\mathrm{AF}}$ the minimum value, measured at $T=1.5 \mathrm{~K}$ and $H=70 \mathrm{kOe}[$ Fig. 3(d)]. These conditions are chosen to minimize the influences of the spin canting and the spin-flop transition on the susceptibility. Inserting in Eq. (2) the experimental values for $H_{\mathrm{sf}}$ and $\chi_{\mathrm{AF} \perp} \sim \frac{3}{2} \chi_{\mathrm{AF}}$, gives $K \simeq 5.5 \times 10^{6} \mathrm{erg} / \mathrm{cm}^{3}$. The anisotropy field of the bulk akaganéite is then $H_{\mathrm{an}}=K / M_{\mathrm{S}} \simeq 13 \mathrm{kOe}$.

As we show next, the effect of the spin canting on $H_{\mathrm{sf}}$ can be safely neglected. The anisotropy constant can be calculated using the expression provided in Ref. 25,

$$
H_{\mathrm{sf}}=m H_{\mathrm{E}}+\sqrt{\left(m H_{\mathrm{E}}\right)^{2}+2 H_{\mathrm{an}} H_{\mathrm{E}}}
$$

where $m=M_{\text {unc }} / M_{\mathrm{S}}=2 \times 10^{-4}$ using the values of the uncompensated magnetization arising from the spin canting (see above). We then obtain $K \simeq 5.6 \times 10^{6} \mathrm{erg} / \mathrm{cm}^{3}$, that is, the same value obtained for the perfect antiferromagnetic structure.

Let us mention that a recent study of akaganéite nanoparticles ${ }^{26}$ reports $K=2.1 \times 10^{4} \mathrm{erg} / \mathrm{cm}^{3}$, much smaller than the anisotropy constant we find here. In that work, $K$ was estimated from the magnetic relaxation rates. These rates were determined by the analysis of Mössbauer spectra measured between 210 and $260 \mathrm{~K}$. However, at these temperatures, close to $T_{\mathrm{N}}$, the magnetic anisotropy can be significantly reduced by thermal fluctuations. Also, reversal magnetization modes other than the coherent rotation of the magnetic moment can contribute, or even dominate, the relaxation in elongated 

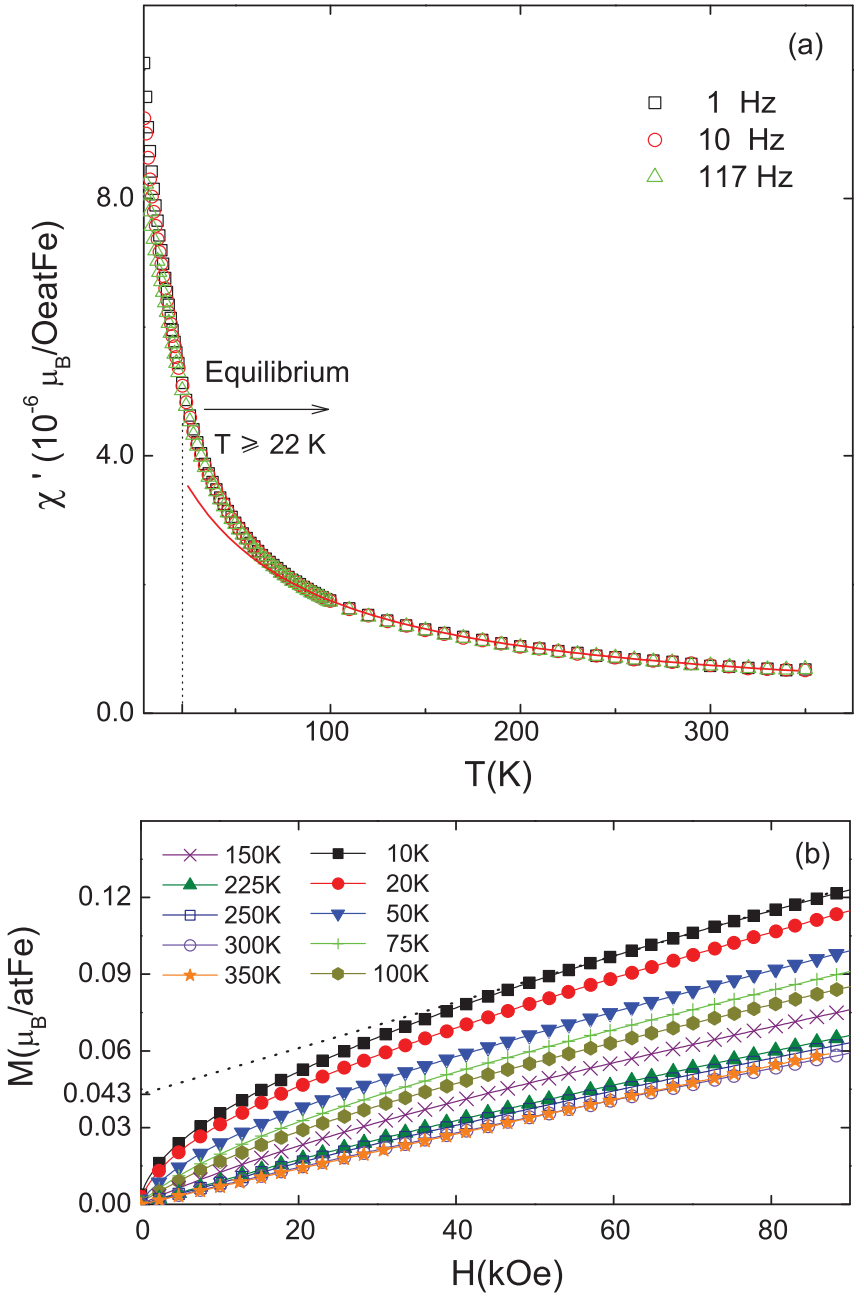

FIG. 4. (Color online) Akaganéite nanoparticles. (a) In-phase ac susceptibility component $\chi^{\prime}$ measured at different frequencies shows that the equilibrium superparamagnetic regime begins at $22 \mathrm{~K}$. The solid line represents the Curie-Weiss law determined above $T_{\mathrm{N}}$. (b) Magnetization vs field curves. The dotted line is a linear fit of the high-field data measured at $T=10 \mathrm{~K}$. The nonlinear magnetization arising from uncompensated magnetic moments becomes dominant for $H \leqslant 50 \mathrm{kOe}$.

nanoparticles. Then, one can still write the activation energy $U$ for the thermally activated relaxation as $U=K V_{\text {eff }}$, but $V_{\text {eff }}$ can be much smaller than the actual particle volume. For this reason, we believe that the value of $K$ that we obtain is more reliable.

\section{Nanoparticles}

The ac susceptibility $\chi^{\prime}$ of akaganéite nanoparticles is shown in Fig. 4(a). Above the Néel temperature $T_{\mathrm{N}}$, it follows the Curie-Weiss law giving $\theta=-49 \pm 13 \mathrm{~K}$ and $\mu_{\text {eff }}=(3.41 \pm 0.08) \mu_{\mathrm{B}}$. Using a gyromagnetic ratio $g=2$ this gives $S=1.28 \pm 0.04$ for the $\mathrm{Fe}^{3+}$ ions. Therefore, we can conclude that the effective atomic spin does not present very large variations with decreasing size. By contrast, $\theta$ is 12 times smaller for the nanoparticles as compared with the bulk. The Néel temperature $T_{\mathrm{N}} \approx 260 \mathrm{~K}$, estimated by heat capacity measurements (not shown), does not show any significant variation with size.

Below $T_{\mathrm{N}}, \chi^{\prime}$ becomes much larger than the AF susceptibility $\chi_{\mathrm{AF}}$ measured on the bulk sample. This reveals the existence of uncompensated spins, as already pointed out by Néel in his seminal papers. ${ }^{1-3,27}$ The susceptibility of a set of AF nanoparticles can then be approximated by the following expression: ${ }^{13,15}$

$$
\chi^{\prime}=\chi_{\mathrm{AF}}+\chi_{\mathrm{unc}}+\chi_{\mathrm{th}}
$$

where $\chi_{\text {unc }}$ and $\chi_{\text {th }}$ are the contributions due to the uncompensated and thermoinduced magnetic moments, respectively. $\chi_{\text {unc }}$ can be expected to show superparamagnetic blocking, associated with the slowing down of the spin reversal, as $T$ decreases. Surprisingly, considering the values of the estimated magnetic anisotropy constant and particle volume, the susceptibility does not show any evidence for such blocking. In fact, above $22 \mathrm{~K}$, in-phase susceptibility $\chi^{\prime}$ curves measured at different frequencies coincide and the out-of-phase susceptibility $\chi^{\prime \prime}$ is smaller than the sensitivity limit, indicating that the nanoparticles behave as superparamagnets in thermal equilibrium. This confirms that rotation modes other than the coherent rotation are contributing to the magnetic relaxation, as discussed in the previous section. This may also account for the lack of proportionality between the energy barrier and the particle volume found by Silva et $a .^{28}$ and for the small value of $K$, compared to the one we obtained here from the spin-flop transition, reported in Ref. 26.

The presence of uncompensated spins also reveals itself in the magnetization curves shown in Fig. 4(b). In contrast with the close-to-linear field dependence observed in bulk akaganéite, the magnetization curves display two contributions. Néel proposed that they can be described as the superposition of the contribution arising from the antiferromagnetic susceptibility plus an additional magnetization due to uncompensated spins. ${ }^{3}$

In order to obtain from these curves the intrinsic $\chi_{\mathrm{AF}}$ in nanoparticles we adopt, as a first approximation, the method developed by Silva et al. ${ }^{12}$ For temperatures above $100 \mathrm{~K}$ and up to $T_{\mathrm{N}}$, the magnetization arising from the uncompensated spins is not yet completely saturated at $90 \mathrm{kOe}$, meaning that $\chi_{\mathrm{AF}}$ cannot be properly determined under these conditions. ${ }^{12}$ Above $T_{\mathrm{N}}$, the magnetization curve is approximately proportional to the applied field and $\chi_{\mathrm{AF}}$ is determined by fitting the magnetization to straight lines. The result is shown in Fig. 5. The antiferromagnetic susceptibility is about 1.5 times larger for the nanoparticles as compared to the bulk, a size effect expected for antiferromagnetic nanoparticles ${ }^{2}$ because of the smaller exchange fields at the surface. This is in agreement with our finding that the Curie-Weiss $\theta$ is also smaller for the nanoparticles. It is worth noticing that $\chi_{\mathrm{AF}}$ is size dependent even at temperatures that are well above $T_{\mathrm{N}}$.

\section{B. Thermoinduced magnetic moment}

We next discuss the origin of the magnetic moment, which, as anticipated in the Introduction, is estimated from the linear 


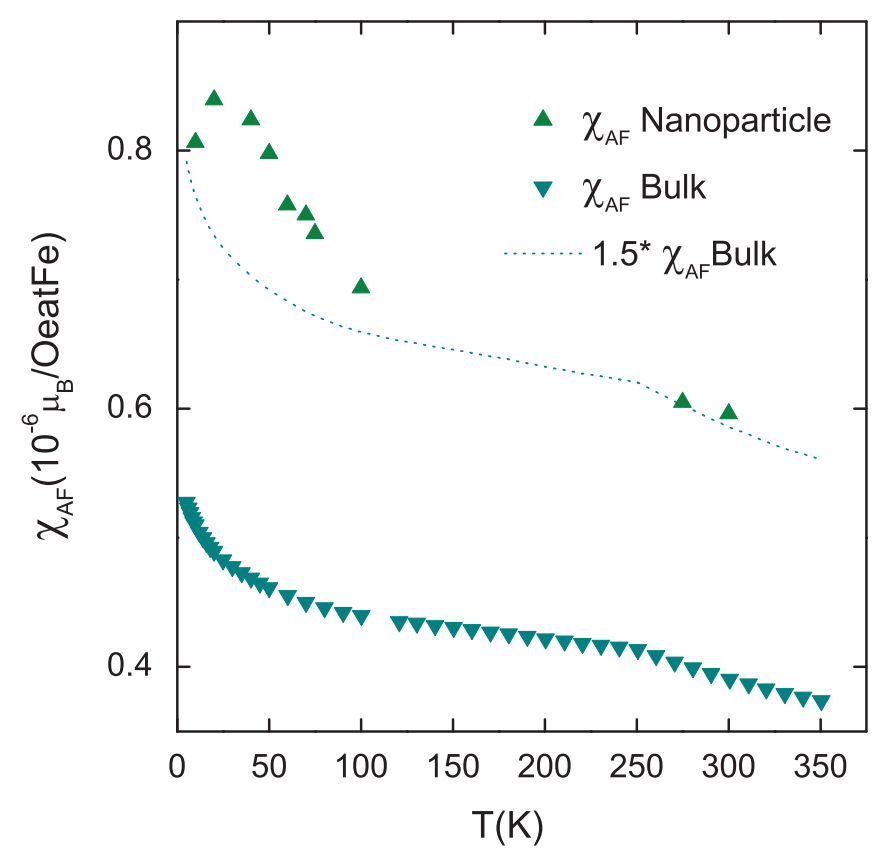

FIG. 5. (Color online) $\chi_{\mathrm{AF}}$ determined from the equilibrium magnetization at high fields for the nanoparticles $(\boldsymbol{\Lambda})$ and bulk samples $(\boldsymbol{\nabla})$, showing that $\chi_{\mathrm{AF}}$ has a similar dependence on temperature in bulk and nanoparticles although it is 1.5 times larger for the latter.

susceptibility. Using Eq. (4) and the expressions provided in Ref. 15 , it is possible to write

$$
\left(\chi^{\prime}-\chi_{\mathrm{AF}}\right) T=n\left[\frac{\mu_{\mathrm{unc}}^{2}}{\mathrm{k}_{\mathrm{B}}}+8 \mathrm{k}_{\mathrm{B}} T^{2}\left(\frac{g \mu_{\mathrm{B}}}{\hbar \omega_{0}}\right)^{2}\right],
$$

where $n$ is the number of particles per akaganéite volume, which can be determined using the size distribution obtained by TEM. The value of the magnetic moment arising from the spin canting can be estimated using the maximum canting angle determined above. It is of the order of $\sim 2 \mu_{\mathrm{B}}$ per particle, and can therefore be safely neglected, as the net magnetic moment per particle exceeds $95 \mu_{\mathrm{B}}$ (see below). In order to extract the thermoinduced contribution, $\chi_{\mathrm{AF}}$ of the nanoparticles was taken, at each temperature, as the value measured on the bulk sample multiplied by 1.5 (see Fig. 5). The quantity $\left(\chi^{\prime}-\chi_{\mathrm{AF}}\right) T$, displayed in Fig. 6, increases from 22 to $50 \mathrm{~K}$ (below $22 \mathrm{~K}, \chi^{\prime}$ deviates from equilibrium and $\chi^{\prime \prime} \neq 0$ ), shows a maximum near $50 \mathrm{~K}$, and then decreases with increasing $T$.

At $T \rightarrow 0$ the thermoinduced contribution should vanish. The uncompensated magnetic moment can be estimated by extrapolating the data measured above $22 \mathrm{~K}$, giving $\mu_{\mathrm{unc}}=$ $(92.04 \pm 0.5) \mu_{\mathrm{B}}$. The relation between $\mu_{\mathrm{unc}}=n_{\mathrm{unc}} S g \mu_{\mathrm{B}}$ and the number of atomic spins in the particle $n$ reflects the origin of the uncompensated magnetic moment in AF nanoparticles. ${ }^{1}$ The number of uncompensated spins $n_{\mathrm{unc}}=\sqrt{n}$ or $n_{\mathrm{unc}}=$ $\sqrt{n_{\text {surf }}}$ for uncompensated spins randomly distributed in the volume or through the surface, respectively. ${ }^{1}$ In spherical nanoparticles $n_{\text {surf }}=n^{2 / 3}$. In elongated nanoparticles $n_{\text {surf }} \simeq$ $N V_{\text {surf }}=N d A$, where $N$ is the number of atoms per unit

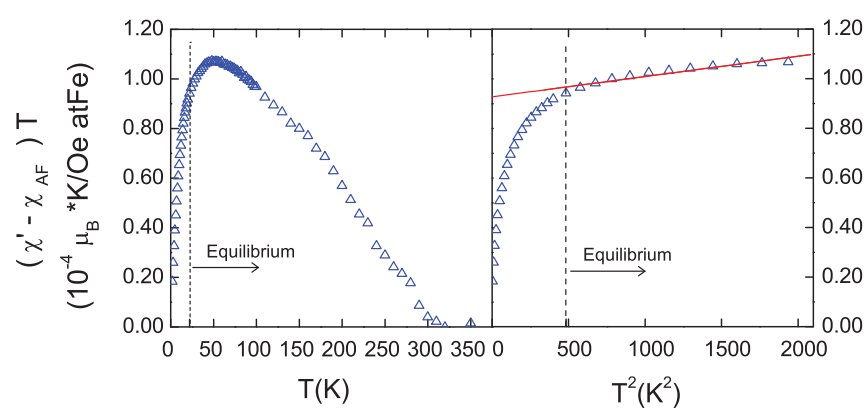

FIG. 6. (Color online) $\left(\chi^{\prime}-\chi_{\mathrm{AF}}\right) T$ of akaganéite nanoparticles; the solid line is a least-squares fit to Eq. (5).

volume and $d$ is the typical thickness of an atomic layer. For akaganéite $N=2.5 \times 10^{22}$ at. $\mathrm{Fe} / \mathrm{cm}^{3}$ and $d \simeq 2.4 \AA$ for the iron atom. $A=\frac{\pi}{2}\left(D^{2}+\frac{D L \alpha}{\sin \alpha}\right)$ is the surface of an ellipsoid with diameter $D$ and length $L$, and $\alpha=\arccos (D / L)$. Nanoparticles with average length $\langle L\rangle=18 \mathrm{~nm}$ and average diameter $\langle D\rangle=5.4 \mathrm{~nm}$ have $n_{\text {surf }}=1375 \mathrm{Fe}$ atoms. This gives $\sqrt{n_{\text {surf }}} S g \mu_{\mathrm{B}}=95 \mu_{\mathrm{B}}$, using the effective spin $S=1.28 \pm$ 0.04 per Fe ion that we found for akaganéite nanoparticles (see Sec. IV A 2), in very good agreement with the value obtained from the experiment.

Let us notice that $\mu_{\text {unc }}$ contributes significantly to the susceptibility [see Eq. (5)] following a Curie dependence with temperature in the superparamagnetic regime. ${ }^{15}$ When this contribution is larger than the contribution due to the thermoinduced magnetic moment the susceptibility decreases monotonically with increasing $T$. This is clearly our case, as shown in Fig. 4(a). Although the magnetic moment increases from 22 to $50 \mathrm{~K}$, the susceptibility still decreases as $T$ increases.

Figure 6 shows that $\left(\chi^{\prime}-\chi_{\mathrm{AF}}\right) T$ becomes approximately proportional to $T^{2}$, as predicted by Eq. (5), in the temperature region $(T \gtrsim 22 \mathrm{~K})$ where $\chi^{\prime}$ provides the equilibrium response. ${ }^{15}$ The fit gives $\hbar \omega_{0}=6.5 \pm 0.4 \mathrm{~K}$. This excitation energy obtained for the nanoparticles can be compared with the spin-wave energy modes of bulk akaganéite. The energy of the lowest-lying excitation mode, with $k=0$ at $H \rightarrow 0$, is approximately given by ${ }^{8,9}$

$$
\hbar \omega_{0}=g \mu_{\mathrm{B}}\left\{H_{\mathrm{an}}\left(2 H_{\mathrm{E}}+H_{\mathrm{an}}\right)\right\}^{1 / 2} .
$$

Use of $H_{\mathrm{an}}$ and $H_{\mathrm{E}}$ values determined above for bulk akaganéite gives $\hbar \omega_{0}=31.5 \mathrm{~K}$. This energy is nearly four times larger than the value determined for the nanoparticles.

The discrepancy can be partly accounted for by the fact that Eq. (6) does not properly describe the elemental magnetic excitations of akaganéite nanoparticles. Equation (6) has been calculated for a perfectly compensated antiferromagnet in which the spontaneous magnetization vanishes at $T=0$. In nanoparticles, the existence of an uncompensated magnetic moment $\mu_{\text {unc }}$ associated with finite size effects influences also the spectrum of spin-wave excitations. This effect was described in Refs. 15 and 29. The energy of the uniform mode can then be calculated using the following expression, ${ }^{29}$ which 
generalizes Eq. (6) to the case where $\mu_{\text {unc }} \neq 0$ :

$$
\hbar \omega_{0}=g \mu_{\mathrm{B}}\left[ \pm \frac{H_{\mathrm{E}}}{2}(\zeta-1)+\sqrt{2 H_{\mathrm{E}} H_{\mathrm{an}}+H_{\mathrm{E}} H_{\mathrm{an}}(\zeta-1)+\left(\frac{H_{\mathrm{E}}}{2}(\zeta-1)\right)^{2}+H_{\mathrm{an}}^{2}}\right]
$$

where $\zeta-1=\mu_{\text {unc }} / M_{\mathrm{S}} V$. Inserting in (7) the uncompensated magnetic moment $\mu_{\mathrm{unc}}=92.04 \mu_{\mathrm{B}}$ determined above for the nanoparticles gives $\hbar \omega_{0,+}=32.8 \mathrm{~K}$ and $\hbar \omega_{0,-}=30.4 \mathrm{~K}$ for the upper and lower $k=0$ modes, respectively. These values are still about four times larger than $\hbar \omega_{0}$ of the nanoparticles, meaning that the presence of an uncompensated magnetic moment cannot account, by itself, for this discrepancy.

As an alternative explanation, the values of $H_{\mathrm{E}}$ and $H_{\mathrm{an}}$ that determine the energy of spin-wave excitations can depend on size. This possibility is supported by the fact, mentioned above, that the Weiss temperature of the nanoparticles, estimated from the paramagnetic susceptibility above $T_{\mathrm{N}}$, is significantly reduced with respect to that of the bulk sample. In addition, energy excitations other than homogeneous spin waves might play a role in nanoparticles. For instance, the local excitation of single spins can be enhanced by the presence of broken exchange bonds at the nanoparticle's surface.

\section{CONCLUSIONS}

Summarizing, we have experimentally determined the exchange $H_{\mathrm{E}}=2.1 \times 10^{6}$ Oe and the anisotropy $H_{\mathrm{an}}=$ $1.3 \times 10^{4}$ Oe fields of bulk akaganéite. The antiferromagnetic susceptibilities $\chi_{\mathrm{AF}}$ of the bulk sample and the nanoparticles show a similar dependence with temperature, though $\chi_{\mathrm{AF}}$ is 1.5 times larger for the latter. We also find that the magnetic moment of akaganéite nanoparticles increases with temperature up to $50 \mathrm{~K}$, decreasing for higher temperatures and nearly vanishing at $T_{\mathrm{N}}=260 \mathrm{~K}$. This behavior provides evidence for the existence of a thermoinduced magnetic moment. The excitation energy that sets the onset of this moment is four times smaller than the energy of homogeneous spin waves in bulk akaganéite, suggesting that such elementary excitations are also strongly affected by size.

\section{ACKNOWLEDGMENTS}

The authors acknowledge N. J. O. Silva for his assistance with the high-field measurements and for fruitful discussions. This work has been funded by the Spanish MICINN and FEDER, Projects No. MAT2007-61621 and No. MAT2009-13977-C03 (MOLCHIP), Project No. CSD 2007-00010 (CONSOLIDER), and DGA Project NABISUP. A.U. acknowledges the financial support provided by contract from the EC NoE "MAGMANET." Part of this work has been supported by EuroMagNET under the EU Contract No. RII3-CT-2004-506239.

\footnotetext{
*fluis@unizar.es

${ }^{1}$ L. Néel, C. R. Acad. Sci. 252, 4075 (1961).

${ }^{2}$ L. Néel, C. R. Acad. Sci. 253, 203 (1961).

${ }^{3}$ L. Néel, C. R. Acad. Sci. 253, 1286 (1961).

${ }^{4}$ S. A. Makhlouf, F. T. Parker, and A. E. Berkowitz, Phys. Rev. B 55, R14717 (1997).

${ }^{5}$ N. J. O. Silva, A. Millán, F. Palacio, E. Kampert, U. Zeitler, H. Rakoto, and V. S. Amaral, Phys. Rev. B 79, 104405 (2009).

${ }^{6}$ S. Kilcoyne and R. Cywinski, J. Magn. Magn. Mater 140-144, 1466 (1995).

${ }^{7}$ F. Luis, E. del Barco, J. M. Hernández, E. Remiro, J. Bartolomé, and J. Tejada, Phys. Rev. B 59, 11837 (1999); N. J. O. Silva, V. S. Amaral, L. D. Carlos, B. Rodríguez-González, L. M. Liz-Marzán, A. Millán, F. Palacio, and V. D. Z. Bermúdez, J. Appl. Phys. 100, 054301 (2006).

${ }^{8}$ S. Mørup and C. Frandsen, Phys. Rev. Lett. 92, 217201 (2004); S. Mørup and B. R. Hansen, Phys. Rev. B 72, 024418 (2005).

${ }^{9}$ D. H. Martin, Magnetism in Solids, 1st ed. (Iliffe Books, London, 1967).

${ }^{10}$ F. Keffer and C. Kittel, Phys. Rev. 85, 329 (1952).

${ }^{11}$ J. G. E. Harris, J. E. Grimaldi, D. D. Awschalom, A. Chiolero, and D. Loss, Phys. Rev. B 60, 3453 (1999); M. S. Seehra, V. S. Babu, A. Manivannan, and J. W. Lynn, ibid. 61, 3513 (2000).
}

${ }^{12}$ N. J. O. Silva, V. S. Amaral, and L. D. Carlos, Phys. Rev. B 71, 184408 (2005).

${ }^{13}$ D. E. Madsen, S. Mørup, and M. F. Hansen, J. Magn. Magn. Mater. 305, 95 (2006)

${ }^{14}$ J. L. García-Palacios, Adv. Chem. Phys. 112, 1 (2000); J. L. GarcíaPalacios, J. B. Gong, and F. Luis, J. Phys. Condens. Matter 21, 456006 (2009).

${ }^{15}$ D. E. Madsen and S. Mørup, Phys. Rev. B 74, 014405 (2006).

${ }^{16}$ J. E. Post and V. F. Buchwald, American Mineralogist 76, 272 (1991); J. E. Post, P. J. Heaney, R. B. V. Dreele, and J. C. Hanson, ibid. 88, 782 (2003).

${ }^{17}$ D. G. Chambaere and E. De Grave, J. Magn. Magn. Mater. 42, 263 (1984).

${ }^{18}$ A. Szytula, M. Balanda, and Z. Dimitrijevic, Phys. Status Solidi A 3, 1033 (1970).

${ }^{19}$ N. Yamamoto, T. Shinjo, M. Kiyama, Y. Bando, and T. Takada, J. Phys. Soc. Jpn. 25, 1267 (1968).

${ }^{20}$ A. Millán, A. Urtizberea, E. Natividad, F. Luis, N. J. O. Silva, F. Palacio, I. Mayoral, M. L. Ruiz-González, J. M. González-Calbet, P. Lecante, and V. Serin, Polymer 50, 1088 (2009).

${ }^{21}$ J. A. A. J. Perenboom, S. A. J. Wiegers, P. C. M. Christianen, U. Zeitler, and J. C. Maan, J. Low Temp. Phys. 133, 181 (2003). 
${ }^{22}$ R. M. Cornell and U. Schwertmann, The Iron Oxides: Structure, Properties, Reactions, Occurences and Uses (Wiley, New York, 2003).

${ }^{23}$ D. S. Kulgawczuk, Z. Obuszko, and A. Szytula, Phys. Status Solidi 26, K83 (1968).

${ }^{24}$ C. A. Barrero, K. E. García, A. L. Morales, S. Kodjikian, and J. M. Greneche, J. Phys. Condens. Matter 18, 6827 (2006).

${ }^{25}$ J. M. D. Coey, A. Barry, J. M. Brotto, H. Rakoto, S. Brennan, W. N. Mussel, A. Collomb, and D. Fruchart, J. Phys. Condens. Matter 7, 759 (1995).
${ }^{26}$ J. Takagi, S. Takakura, T. Okada, T. Kobayashi, M. Ozaki, H. Kihira, and T. Mizoguchi, Corros. Sci. 50, 1971 (2008).

${ }^{27}$ L. Néel, C. R. Acad. Sci. 253, 9 (1961).

${ }^{28}$ N. J. O. Silva, V. S. Amaral, L. D. Carlos, B. Rodríguez-González, L. M. Liz-Marzán, T. S. Berquó, S. K. Banerjee, V. de Zea Bermúdez, A. Millán, and F. Palacio, Phys. Rev. B 77, 134426 (2008).

${ }^{29}$ C. R. H. Bahl, J. Garde, K. Lefmann, T. B. S. Jensen, P. A. Lindgard, D. E. Madsen, and S. Mørup, Eur. Phys. J. B 62, 53 (2008). 